\title{
A STRICT POSITIVSTELLENSATZ FOR RINGS OF DEFINABLE ANALYTIC FUNCTIONS
}

\author{
ANDREAS FISCHER \\ (Communicated by Julia Knight)
}

\begin{abstract}
Consider an expansion of the real field in which every unary definable continuous function can be ultimately majorized by a definable analytic function. We prove the strict Positivstellensatz for analytic functions which are definable in such structures. The methods also work for a large class of quasianalytic subrings of the ring of those smooth functions that are definable in a polynomially bounded structure.
\end{abstract}

\section{INTRODUCTION}

Let $k$ be any nonnegative integer. Krivine's result (cf. 9]), often referred to as Stengle's Positivstellensatz (cf. [15]), characterizes the polynomials nonnegative on the basic closed semialgebraic set

$$
F:=\left\{f_{1} \geq 0, \ldots, f_{k} \geq 0\right\}
$$

as follows:

A polynomial $g$ is nonnegative on $F$ if and only if there are polynomials $p_{1}, p_{2}$ in the positive cone $C$ generated by the polynomials $f_{i}$ and the sums of squares such that

$$
p_{1} g=p_{2}+g^{2 m}
$$

for some $m \geq 0$. The polynomial is strictly positive on $F$ if and only if there is an equation

$$
p_{1} g=1+p_{2},
$$

where $p_{1}, p_{2} \in C$; see for example [5. Cor. 4.2.10].

Schmüdgen proved in [13] that if $F$ is compact and $g$ strictly positive on $F$, then $p_{1}$ is not needed, and Putinar verified additional conditions on the $f_{i}$ for $g$ belonging to the quadratic module generated by the $f_{i}$.

In [1, Acquistapace, Andradas and Broglia proved the strict Positivstellensatz for (global) analytic functions on Euclidean spaces.

Here we generalize and strengthen the result in 1 by providing a proof which, among other things, also preserves definability.

Received by the editors April 21, 2009 and, in revised form, May 1, 2010, February 7, 2011, and July 12, 2011.

2010 Mathematics Subject Classification. Primary 03C64, 14P10; Secondary 13J30, 26 E10.

Key words and phrases. Positivstellensatz, analytic and smooth function, polynomially bounded structure.

The author is a postdoctoral fellow of the Thematic Program on o-minimal Structures and Real Analytic Geometry at the Fields Institute. 
Let $\mathfrak{R}$ denote an expansion of the real field (see [4, 6, 7] for introductions to structures over the real field). By definable, we always mean definable in $\mathfrak{R}$ with parameters from $\mathbb{R}$, and functions are definable if their graphs are definable.

We consider structures $\mathfrak{R}$ which satisfy the following condition:

(A) For every definable continuous function $\phi:[0, \infty) \rightarrow \mathbb{R}$ there is a definable analytic function $\Phi:[-1, \infty) \rightarrow \mathbb{R}$ such that $\Phi \geq \phi$ on $[0, \infty)$.

This property is not very restrictive. No structure failing this property is known to the author; in particular, every known o-minimal expansion of the real field satisfies this property. Moreover, the structure in which all subsets of Euclidean spaces are definable satisfies this property.

For a finite set of functions $f_{1}, \ldots, f_{k}: \mathbb{R}^{n} \rightarrow \mathbb{R}$, we let

$$
F:=\bigcap_{i=1}^{k}\left\{f_{i} \geq 0\right\} .
$$

We shall prove the following theorem.

Theorem 1.1. Assume that $\mathfrak{R}$ satisfies property $(A)$. Let $g, f_{1}, \ldots, f_{k}: \mathbb{R}^{n} \rightarrow \mathbb{R}$ be definable and analytic such that $g>0$ on $F$. Then there are definable analytic functions $v_{0}, \ldots, v_{k} \in \mathcal{C}^{\omega}\left(\mathbb{R}^{n},(0, \infty)\right)$ such that

$$
g=v_{0}^{2}+\sum_{i=1}^{k} v_{i}^{2} f_{i} .
$$

Denote by $\mathcal{C}_{\text {def }}^{\infty}\left(\mathbb{R}^{n}, \mathbb{R}\right)$ the definable smooth functions from $\mathbb{R}^{n}$ to $\mathbb{R}$. Suppose now that $\mathfrak{R}$ is additionally polynomially bounded; that is, every definable continuous function $\phi:[0, \infty) \rightarrow \mathbb{R}$ is bounded by some polynomial. Then the ring $\mathcal{C}_{\text {def }}^{\infty}\left(\mathbb{R}^{n}, \mathbb{R}\right)$ is quasianalytic; i.e., the Taylor homomorphism

$$
T: \mathcal{C}_{\text {def }}^{\infty}\left(\mathbb{R}^{n}, \mathbb{R}\right) \rightarrow \mathbb{R}\left[\left[X_{1}, \ldots, X_{n}\right]\right]
$$

mapping $f$ to its Taylor series at 0 is injective (cf. [10]).

In particular, every subring of $\mathcal{C}_{\text {def }}^{\infty}\left(\mathbb{R}^{n}, \mathbb{R}\right)$ is quasianalytic. However, smooth and analytic do not always coincide in polynomially bounded o-minimal structures; see 12. For a certain class of such quasianalytic rings, we also have the strict Positivstellensatz. This Positivstellensatz can be formulated in a more general form as follows.

Theorem 1.2. Let $\mathfrak{R}$ be a polynomially bounded o-minimal expansion of the real field. Suppose $\mathcal{B}$ is a subring of $\mathcal{C}_{\text {def }}^{\infty}\left(\mathbb{R}^{n}, \mathbb{R}\right)$ such that

(a) $\mathbb{R}\left[X_{1}, \ldots, X_{n}\right] \subset \mathcal{B}$;

(b) if $f \in \mathcal{B}$, and $f>0$, then $1 / f$ and $\sqrt{f}$ belong to $\mathcal{B}$.

Let $g, f_{1}, \ldots, f_{k} \in \mathcal{B}$ such that $g>0$ on $F$. Then there are strictly positive functions $v_{0}, \ldots, v_{k} \in \mathcal{B}$ such that

$$
g=v_{0}^{2}+\sum_{i=1}^{k} v_{i}^{2} f_{i} .
$$

The ring $\mathcal{C}_{\text {def }}^{\omega}\left(\mathbb{R}^{n}, \mathbb{R}\right)$ is an example of such a ring $\mathcal{B}$.

Remark 1.3. If one considers differentiable or smooth functions when the exponential function is definable, then one obtains a far stronger Positivstellensatz; see [2, 8. 
The proofs in [1] essentially use the fairly transcendental tool of analytic approximation of continuous functions with respect to the (strong) Whitney topology. So far, definable analytic approximation is only known for the semialgebraic structure; cf. [14. By giving a rather explicit construction of the functions $v_{0}, \ldots, v_{k}$ of the above theorems we avoid approximation while preserving definability. Section 2 is devoted to the construction of these functions to prove both Theorem 1.1 and Theorem 1.2. In the final section, Section 3, we briefly discuss some generalizations and a consequence of our theorems.

\section{Proofs}

2.1. Two lemmas. We use $\|\cdot\|$ to denote the Euclidean norm, and $|\cdot|$ to denote the absolute value. We start by proving two elementary lemmas.

Lemma 2.1. Let $\varepsilon: \mathbb{R}^{n} \rightarrow(0, \infty)$ be definable and continuous.

(a) If $\mathfrak{R}$ satisfies $(A)$, then there is a definable $\mathcal{C}^{\omega}$ function $\rho: \mathbb{R}^{n} \rightarrow(0,1)$ such that $\rho<\varepsilon$ on $\mathbb{R}^{n}$.

(b) If $\mathfrak{R}$ is polynomially bounded, then there is a strictly positive function $\rho \in \mathcal{B}$ such that $\rho<\varepsilon$ on $\mathbb{R}^{n}$.

Proof. For $t \geq 0$ let

$$
L(t):=\sup \left\{\frac{1}{\varepsilon(x)}:\|x\| \leq \sqrt{t}\right\} .
$$

To prove (a), we notice that by (A) there is a definable function $\Phi:[-1, \infty) \rightarrow \mathbb{R}$ with $\Phi(t)>L(t)$. Define $\rho$ by

$$
\rho=1 /\left(1+\Phi\left(\|x\|^{2}\right)\right) .
$$

To prove (b), we observe that the polynomial boundedness of $\Re$ implies that there is a polynomial $p$ such that $p(t)>L(t)$. Set $\rho:=1 /\left(1+p\left(\|x\|^{2}\right)\right)$.

Lemma 2.2. Let $U \subset \mathbb{R}^{n}$ be a definable open set, and let $B \subset U$ be a definable closed set. Let $\varepsilon: U \rightarrow(0, \infty)$ be a definable continuous function. Then there exists a definable continuous function $\tilde{\varepsilon}: \mathbb{R}^{n} \rightarrow(0, \infty)$ such that $\tilde{\varepsilon}=\varepsilon$ on $B$.

Proof. The case $B=\emptyset$ is trivial, so we may assume that $B \neq \emptyset$. We may also assume that $U \neq \mathbb{R}^{n}$; otherwise one can take $\tilde{\varepsilon}=\varepsilon$. Let

$$
V=\left\{x \in \mathbb{R}^{n}: \operatorname{dist}(x, B) \geq \operatorname{dist}\left(x, \mathbb{R}^{n} \backslash U\right)\right\} .
$$

Let $\phi_{1}(x)=\operatorname{dist}(x, B)$, and let $\phi_{2}(x)=\operatorname{dist}(x, V)$. Then

$$
\tilde{\varepsilon}:=\frac{\phi_{1}+\varepsilon \phi_{2}}{\phi_{1}+\phi_{2}}
$$

satisfies the required properties.

2.2. Reduction step. The proofs of Theorem 1.1 and Theorem 1.2 are similar. So if $\mathfrak{R}$ is an expansion of $\mathbb{R}$ which is not polynomially bounded but satisfies (A), we let $\mathcal{A}:=\mathcal{C}_{\text {def }}^{\omega}\left(\mathbb{R}^{n}, \mathbb{R}\right)$, and if $\Re$ is a polynomially bounded expansion of the real field, we let $\mathcal{A}$ be a subring $\mathcal{B}$ of $\mathcal{C}_{\text {def }}^{\infty}\left(\mathbb{R}^{n}, \mathbb{R}\right)$ satisfying the conditions (a) and (b) of Theorem 1.2 .

Moreover, the theorems are trivial if $k=0$. So from now on, we assume $k \geq 1$.

The following lemma is used to reduce the proof of the theorems to the case $k=1$. 
Lemma 2.3. Let $g, f_{1}, \ldots, f_{k} \in \mathcal{A}$ and let $g>0$ on $F$. Then there exist strictly positive functions $s_{1}, \ldots, s_{k} \in \mathcal{A}$ such that

$$
h:=\sum_{i=1}^{k} s_{i}^{2} f_{i}
$$

satisfies

$$
F \subset\{h \geq 0\} \subset\{g>0\} .
$$

Proof. The case $F=\mathbb{R}^{n}$ is trivial, so we may assume that $F \neq \mathbb{R}^{n}$.

Consider the function $\varepsilon: \mathbb{R}^{n} \backslash F \rightarrow \mathbb{R}$ defined by

$$
\varepsilon(x):=\frac{\sum_{i=1}^{k} \max \left(-f_{i}(x), 0\right)^{2}}{\sum_{i=1}^{k}\left|f_{i}(x)\right|} .
$$

This function is definable, continuous and strictly positive (particularly on $\{g \leq 0\}$ ). By Lemma 2.2. there is a definable continuous function $\tilde{\varepsilon}: \mathbb{R}^{n} \rightarrow(0, \infty)$ such that $\tilde{\varepsilon}=\varepsilon$ on $\{g \leq 0\}$. By Lemma 2.1, there is a $\rho \in \mathcal{A}$ such that $0<\rho<\tilde{\varepsilon}$.

For $i=1, \ldots, k$, set

$$
\phi_{i}:=\frac{1}{2}\left(\sqrt{\rho^{2}+f_{i}^{2}}-f_{i}\right) .
$$

Then we have

$$
0<\phi_{i} \leq \frac{\rho}{2} \text { on }\left\{f_{i} \geq 0\right\}
$$

and

$$
\left|f_{i}\right|<\phi_{i} \leq\left|f_{i}\right|+\frac{\rho}{2} \text { on }\left\{f_{i} \leq 0\right\} .
$$

We notice that the functions $\phi_{i}$ are strictly positive. Hence, there are strictly positive functions $s_{i} \in \mathcal{A}$ such that $s_{i}^{2}=\phi_{i}$. Define

$$
h:=\sum_{i=1}^{k} s_{i}^{2} f_{i} .
$$

Then $F \subset\{h \geq 0\}$. Let $\delta_{i}$ be the characteristic function of the set $\left\{f_{i}>0\right\}$. Then we have on $\{g \leq 0\}$ that

$$
\begin{aligned}
h & =\sum_{i=1}^{k} \phi_{i} f_{i} \\
& \leq \sum_{i=1}^{k}\left(\max \left(-f_{i}, 0\right)+\delta_{i} \rho / 2\right) f_{i} \\
& \leq \sum_{i=1}^{k} \max \left(-f_{i}, 0\right) f_{i}+\frac{\rho}{2} \sum_{i=1}^{k}\left|f_{i}\right| \\
& \leq-\sum_{i=1}^{k} \max \left(-f_{i}, 0\right)^{2}+\frac{1}{2} \sum_{i=1}^{k} \max \left(-f_{i}, 0\right)^{2} \\
& =\frac{-1}{2} \sum_{i=1}^{k} \max \left(-f_{i}, 0\right)^{2} \\
& <0 .
\end{aligned}
$$

Hence $\{h \geq 0\} \subset\{g>0\}$. 
2.3. Proof of Theorem 1.1 and Theorem 1.2. We are now able to prove the theorems. By convention, we set $a / 0:=+\infty$ if $a \in(0, \infty)$.

Proof. By Lemma 2.3 there are strictly positive functions $s_{1}, \ldots, s_{k} \in \mathcal{A}$ such that

$$
h=\sum_{i=1}^{k} s_{i}^{2} f_{i}
$$

satisfies

$$
F \subset\{h \geq 0\} \subset\{g>0\}
$$

Hence

$$
\{h \geq 0\} \cap\{g \leq 0\}=\emptyset .
$$

Define $\varepsilon:\{h \geq 0\} \cup\{g \leq 0\} \rightarrow \mathbb{R}$ by

$$
\varepsilon(x):= \begin{cases}\min \left(1, g(x)+h(x), \frac{g(x)}{\sqrt{|h(x)|}}, \frac{g(x)^{2}}{2|h(x)(g(x)+h(x))|}\right) & \text { for } x \in\{h \geq 0\}, \\ \min \left(1, \frac{-h(x)}{2}, \frac{h(x)^{2}}{2|g(x)|}\right) & \text { for } x \in\{g \leq 0\} .\end{cases}
$$

Then $\varepsilon$ is continuous and strictly positive, and, by the same definition, it can be extended to a continuous, strictly positive function $\varepsilon^{\prime}$ on some open definable neighbourhood $U$ of $\{h \geq 0\} \cup\{g \leq 0\}$. So $\varepsilon$ extends to a definable, continuous, strictly positive function on $\mathbb{R}^{n}$ by Lemma 2.2. By Lemma 2.1, there is a strictly positive $\rho \in \mathcal{A}$ which is majorized by the extended function $\varepsilon$.

Define the functions $\sigma_{1}, \sigma_{2}, \tau_{1}$ and $\tau_{2}$ by

$$
\begin{aligned}
\tau_{1} & :=\sqrt{\rho^{2}+(g+h)^{2}}, \\
\tau_{2} & :=\sqrt{\rho^{2}+g^{2}}, \\
\sigma_{1} & :=\frac{1}{2}\left(\sqrt{\rho^{4}+g^{2}}+g\right), \\
\sigma_{2} & :=\frac{1}{2}\left(\sqrt{\rho^{4}+(g+h)^{2}}-(g+h)\right) .
\end{aligned}
$$

Set

$$
w:=\frac{\sigma_{1}}{\tau_{1}}+\frac{\sigma_{2}}{\tau_{2}} .
$$

This function is strictly positive and belongs to $\mathcal{A}$.

We claim that the function $u$ given by

$$
u:=g-w h
$$

is strictly positive. This will be proved in several steps.

Step 1. Observe that on $\{g \leq 0\}$ we have

$$
\frac{\sigma_{1}}{\tau_{1}} \leq \frac{\frac{1}{2} \rho^{2}}{\sqrt{\rho^{2}+(g+h)^{2}}} \leq \frac{\rho}{2},
$$

and on $\{h \geq 0\}$ we have

$$
\frac{\sigma_{2}}{\tau_{2}} \leq \frac{\frac{1}{2} \rho^{2}}{\sqrt{\rho^{2}+h^{2}}} \leq \frac{\rho}{2}
$$


Step 2 (a). On $\{h \geq 0\}$ we have $g>0$, so

$$
\begin{aligned}
g(x) \tau_{1}(x) & -h(x) \sigma_{1}(x) \\
& =g(x) \sqrt{\rho(x)^{2}+(g(x)+h(x))^{2}}-\frac{h(x)}{2}\left(\sqrt{g(x)^{2}+\rho(x)^{4}}+g(x)\right) \\
& \geq g(x) \sqrt{\rho(x)^{2}+(g(x)+h(x))^{2}}-h(x)\left(g(x)+\frac{\rho(x)^{2}}{2}\right) \\
& >g(x)(g(x)+h(x))-h(x) g(x)-\frac{1}{2} h(x) \min \left(1, \frac{g(x)^{2}}{|h(x)|}\right) \\
& \geq \frac{g(x)^{2}}{2} .
\end{aligned}
$$

Hence, the function $\varepsilon_{1}:\{h \geq 0\} \rightarrow \mathbb{R}$ defined by

$$
\varepsilon_{1}:=\frac{g \tau_{1}-h \sigma_{1}}{\tau_{1}}>\frac{g^{2}}{2 \sqrt{\rho^{2}+(g+h)^{2}}} \geq \frac{g^{2}}{2 \sqrt{2}(g+h)}
$$

is strictly positive.

Step 2 (b). On $\{g \leq 0\}$ we have $h<0$, so

$$
\begin{aligned}
g \tau_{2}-h \sigma_{2} & =g \sqrt{\rho^{2}+h^{2}}-h \frac{1}{2}\left(\sqrt{(g+h)^{2}+\rho^{4}}-(g+h)\right) \\
& >g \sqrt{\rho^{2}+h^{2}}-h(-(g+h)) \\
& >-g h+\rho g+h g+h^{2} \\
& \geq \frac{h^{2}}{2} .
\end{aligned}
$$

Hence, the function $\varepsilon_{2}:\{g \leq 0\} \rightarrow \mathbb{R}$ defined by

$$
\varepsilon_{2}:=\frac{\tau_{2} g-\sigma_{2} h}{\tau_{2}}>\frac{h^{2}}{2 \sqrt{\rho^{2}+h^{2}}}
$$

is strictly positive.

Step 3. Verifying $u>0$.

On $\{h \geq 0\}$ we have

$$
\begin{aligned}
u(x) & =g(x)-\frac{\sigma_{1}(x)}{\tau_{1}(x)} h(x)-\frac{\sigma_{2}(x)}{\tau_{2}(x)} h(x) \\
& \geq \varepsilon_{1}(x)-\frac{\rho(x)}{2} h(x) \\
& \geq \frac{g(x)^{2}}{2 \sqrt{2}(h(x)+g(x))}-\frac{1}{2} \min \left(1, \frac{g(x)^{2}}{2|h(x)(g(x)+h(x))|}\right) h(x) \\
& \geq\left(\frac{1}{2 \sqrt{2}}-\frac{1}{4}\right) \frac{g(x)^{2}}{h(x)+g(x)}>0 .
\end{aligned}
$$

On $\{g \leq 0\}$ we have

$$
u=g-\frac{\sigma_{1}}{\tau_{1}} h-\frac{\sigma_{2}}{\tau_{2}} h=\varepsilon_{2}-\frac{\sigma_{1}}{\tau_{1}} h>0,
$$

since $h<0$ on $\{g \leq 0\}$. 
On $\{g>0\} \cap\{h<0\}$ we evidently have $u=g-w h>0$.

Finishing the proof of the theorems, we set $v_{0}=\sqrt{u}$ and $v_{i}=\sqrt{w} s_{i}$ and obtain the equality

$$
g=u+w h=v_{0}^{2}+\sqrt{w}^{2} \sum_{i=1}^{k} s_{i}^{2} f_{i}=v_{0}^{2}+\sum_{i=1}^{k} v_{i}^{2} f_{i} .
$$

\section{REMARKS AND CONSEQUENCES}

Remark 3.1. Theorem 1.2 remains true if we replace $\mathbb{R}$ by any real closed field $R$, and $\mathfrak{R}$ by any polynomially bounded definably complete expansion of $R$; cf. [11].

Remark 3.2. The proofs do not make use of the quasianalyticity of the considered rings. They actually work for more general rings, as long as a corresponding statement of Lemma 2.1 holds true. Lemma 2.2 works (in an appropriate formulation) for continuous functions on a Banach space.

Remark 3.3. Let $d \in \mathbb{N}$. Suppose one stipulates that the $\operatorname{ring} \mathcal{A}$ additionally satisfies the following property: If $f \in \mathcal{A}$ and $f>0$, then $f^{1 /(2 d)} \in \mathcal{A}$. Then one can write

$$
g=v_{0}^{2 d}+\sum_{i=1}^{k} v_{i}^{2 d} f_{i}
$$

in the statement of Theorem [1.2. For Theorem [1.1. this property is evidently satisfied.

Let $\operatorname{Spec}_{r}(\mathcal{A})$ denote the real spectrum of $\mathcal{A}$; see [3, Chap. 7]. Then $\mathbb{R}^{n}$ embeds canonically into $\operatorname{Spec}_{r}(\mathcal{A})$ by mapping $x$ to the prime cone $\tilde{x}$ consisting of all $f \in \mathcal{A}$ such that $f(x) \geq 0$.

The following corollary is a particular case of the Artin-Lang property.

Corollary 3.4. Let $f_{1}, \ldots, f_{k} \in \mathcal{A}$. Then

$$
\tilde{S}=\left\{\alpha \in \operatorname{Spec}_{r}(\mathcal{A}) ; f_{1}(\alpha) \geq 0, \ldots, f_{k}(\alpha) \geq 0\right\}
$$

is not empty if and only if $S=\mathbb{R}^{n} \cap \tilde{S}$ is not empty.

Proof. Evidently, if $\tilde{S}=\emptyset$, then $S=\emptyset$. Suppose now that

$$
S=\tilde{S} \cap \mathbb{R}^{n}=\left\{x \in \mathbb{R}^{n}: f_{1}(x) \geq 0, \ldots, f_{k}(x) \geq 0\right\}=\emptyset .
$$

Then $-f_{k}$ is strictly positive on the set

$$
F=\left\{x \in \mathbb{R}^{n}: f_{1},(x) \geq 0, \ldots, f_{k-1}(x) \geq 0\right\} .
$$

By Theorem 1.2 and Theorem 1.1, there are strictly positive functions $v_{0}, \ldots, v_{k-1} \in$ $\mathcal{A}$ such that

$$
-f_{k}=v_{0}^{2}+\sum_{i=1}^{k-1} v_{i}^{2} f_{i} .
$$

Set $t_{i}=v_{i} / v_{0}$ for $i=1, \ldots, k-1$ and $t_{k}=1 / v_{0}$. Then

$$
0=1+\sum_{i=1}^{k} t_{i}^{2} f_{i}
$$


Assume, for a contradiction, that there exists a $\beta \in \tilde{S}$. Then $f_{i}(\beta) \geq 0$ and $t_{i}^{2}(\beta) \geq 0$ for all $i$. Hence, we obtain the contradiction

$$
0=1+\sum_{i=1}^{k} t_{i}^{2}(\beta) f_{i}(\beta) \geq 1 .
$$

So $\tilde{S}$ is empty.

\section{ACKNOWLEDGEMENT}

The author would like to thank the anonymous referee for insightful comments.

\section{REFERENCES}

1. F. Acquistapace, C. Andradas, F. Broglia, The strict Positivstellensatz for global analytic functions and the moment problem for semianalytic sets. Math. Ann. 316 (2000), no. 4, 609-616. MR 1758445 (2001g:14087)

2. F. Acquistapace, C. Andradas, F. Broglia, The Positivstellensatz for definable functions on o-minimal structures. Illinois J. Math. 46 (2002), no. 3, 685-693. MR1951235 (2003k:03053)

3. J. Bochnak, M. Coste, M.-F. Roy, Real algebraic geometry. Translated from the 1987 French original. Revised by the authors. Ergebnisse der Mathematik und ihrer Grenzgebiete (3) [Results in Mathematics and Related Areas (3)], 36. Springer-Verlag, Berlin, 1998. x+430 pp. MR1659509 (2000a:14067)

4. M. Coste, An Introduction to O-minimal Geometry. Dip. Mat. Univ. Pisa, Dottorato di Ricerca in Matematica, Istituti Editoriali e Poligrafici Internazionali, Pisa, 2000.

5. A. Prestel, C. N. Delzell, Positive polynomials. From Hilbert's 17th problem to real algebra. Springer Monographs in Mathematics. Berlin: Springer, 2001. viii+268 pp. MR,1829790 (2002k:13044)

6. L. van den Dries, Tame Topology and O-minimal Structures. LMS Lecture Notes 248, Cambridge University Press, 1998. MR1633348 (99j:03001)

7. L. van den Dries, C. Miller, Geometric categories and o-minimal structures. Duke Math. J. 84, no. 2, 497-540 (1996). MR1404337(97i:32008)

8. A. Fischer, Positivstellensätze for differentiable functions, Positivity. DOI: 10.1007/s11117010-0077-5

9. J.-L. Krivine, Anneaux préordonnés, J. Analyse Math. 12, 307-326 (1964). MR0175937 $(31: 213)$

10. C. Miller, Infinite differentiability in polynomially bounded o-minimal structures. Proc. Amer. Math. Soc. 123, no. 8, 2551-2555 (1995). MR1257118(95j:03069)

11. C. Miller, Expansions of dense linear orders with the intermediate value property. J. Symbolic Logic 66 (2001), no. 4, 1783-1790. MR1877021 (2003j:03044)

12. J.-P. Rolin, P. Speissegger, A. J. Wilkie, Quasianalytic Denjoy-Carleman classes and ominimality, J. Amer. Math. Soc. 16 (2003), no. 4, 751-777. MR1992825 (2004g:14065)

13. K. Schmüdgen, The $K$-moment problem for compact semi-algebraic sets. Math. Ann. 289 (1991), no. 2, 203-206. MR.1092173 (92b:44011)

14. M. Shiota, Approximation theorems for Nash mappings and Nash manifolds. Trans. Amer. Math. Soc. 293, no. 1, 319-337 (1986). MR814925 (87e:58004)

15. G. Stengle, A nullstellensatz and a positivstellensatz in semialgebraic geometry. Math. Ann. 207 (1974), 87-97. MR0332747 (48:11073)

Gymnasium St. Ursula, Ursulastrasse 8-10, Dorsten, Germany

Current address: Comenius Gymnasium Datteln, Südring 150, 45711 Datteln, Germany

E-mail address: el.fischerandreas@live.de 